\title{
Flexion Reflex Can Interrupt and Reset the Swimming Rhythm
}

\author{
Matthew S. Elson ${ }^{1}$ and Ari Berkowitz ${ }^{1,2}$ \\ ${ }^{1}$ Department of Biology and ${ }^{2}$ Cellular and Behavioral Neurobiology Graduate Program, University of Oklahoma, Norman, Oklahoma 73019
}

The spinal cord can generate the hip flexor nerve activity underlying leg withdrawal (flexion reflex) and the rhythmic, alternating hip flexor and extensor activities underlying locomotion and scratching, even in the absence of brain inputs and movement-related sensory feedback. It has been hypothesized that a common set of spinal interneurons mediates flexion reflex and the flexion components of locomotion and scratching. Leg cutaneous stimuli that evoke flexion reflex can alter the timing of (i.e., reset) cat walking and turtle scratching rhythms; in addition, reflex responses to leg cutaneous stimuli can be modified during cat and human walking and turtle scratching. Both of these effects depend on the phase (flexion or extension) of the rhythm in which the stimuli occur. However, similar interactions between leg flexion reflex and swimming have not been reported. We show here that a tap to the foot interrupted and reset the rhythm of forward swimming in spinal, immobilized turtles if the tap occurred during the swim hip extensor phase. In addition, the hip flexor nerve response to an electrical foot stimulus was reduced or eliminated during the swim hip extensor phase. These two phasedependent effects of flexion reflex on the swim rhythm and vice versa together demonstrate that the flexion reflex spinal circuit shares key components with or has strong interactions with the swimming spinal network, as has been shown previously for cat walking and turtle scratching. Therefore, leg flexion reflex circuits likely share key spinal interneurons with locomotion and scratching networks across limbed vertebrates generally.

Key words: central pattern generator; locomotion; multifunctional; spinal cord; turtle; withdrawal

\section{Significance Statement}

The spinal cord can generate leg withdrawal (flexion reflex), locomotion, and scratching in limbed vertebrates. It has been hypothesized that there is a common set of spinal cord neurons that produce hip flexion during flexion reflex, locomotion, and scratching based on evidence from studies of cat and human walking and turtle scratching. We show here that flexion reflex and swimming also share key spinal cord components based on evidence from turtles. Foot stimulation can reset the timing of the swimming rhythm and the response to each foot stimulation can itself be altered by the swim rhythm. Collectively, these studies suggest that spinal cord neuronal networks underlying flexion reflex, multiple forms of locomotion, and scratching share key components.

\section{Introduction}

Central pattern generators (CPGs) are networks of neurons in the CNS that can generate the rhythmic neural activity underlying many rhythmic behaviors, including several forms of locomotion

Received Sept. 28, 2015; revised Jan. 29, 2016; accepted Feb. 2, 2016.

Author contributions: M.S.E. and A.B. designed research; M.S.E. performed research; M.S.E. and A.B. analyzed data; M.S.E. and A.B. wrote the paper.

This work was supported by the National Science Foundation (Grants IOB- 0349620 and IOS-0950370) and the Oklahoma Center for the Advancement of Science and Technology (Grant HR13-120 to A.B.), and the Department of Biology of the University of Oklahoma (M. Blanche Adams and M. Frances Adams Scholarship to M.S.E.). We thank Zhao-Zhe Hao for assistance in data analysis and Ingo Schlupp for statistical advice. We thank Zhao-Zhe Hao, Paul

S.G. Stein, and two anonymous reviewers for comments on an earlier version of this manuscript.

The authors declare no competing financial interests.

Correspondence should be addressed to Ari Berkowitz, Department of Biology, University of Oklahoma, 730 Van Vleet Oval, Norman, 0K 73019. E-mail: ari@ou.edu.

DOI:10.1523/JNEUROSCI.3587-15.2016

Copyright $\odot 2016$ the authors $\quad 0270-6474 / 16 / 362819-08 \$ 15.00 / 0$ and scratching (Brown, 1911; Marder and Calabrese, 1996). An ongoing question is to what extent each CPG shares components with other CPGs or with neural circuits that control episodic behaviors (Briggman and Kristan, 2008; Berkowitz et al., 2010). Sherrington $(1906,1910)$ noted the similarity of leg withdrawal (flexion reflex) to the flexion phase of walking in cats and demonstrated strong interactions between flexion reflex and walking. It was later proposed that the sensory neurons that trigger the late component of flexion reflex (flexion reflex afferents) activate common components of the flexion reflex circuit and the walking CPG in cats (Jankowska et al., 1967; Lundberg, 1979; Hultborn, 2006). Evidence has accumulated that spinal cord circuits controlling flexion reflex and walking do share some components (Lundberg, 1979; Burke, 1999; McCrea, 2001; Duysens et al., 2013). Many spinal cord interneurons are activated during the forward swimming rhythm, scratching rhythms, and flexion re- 
flex motor patterns in turtles, consistent with shared circuitry for these three behaviors (Berkowitz, 2010). Other turtle spinal interneurons, however, are activated selectively during flexion reflex motor patterns and are inhibited during both swimming and scratching rhythms (Berkowitz, 2007). Therefore, the extent of sharing of circuitry between flexion reflex and locomotion is still uncertain.

One of the most definitive criteria to show that a neuron is a member of a CPG is the demonstration that brief stimulation of the neuron can disrupt and reset the CPG rhythm such that the timing of the rhythm remains altered well after the perturbing stimulus (Marder and Calabrese, 1996). This criterion, however, usually fails for larger CPGs such as are typical in vertebrates, presumably because each neuron makes only a small contribution to such a large network. However, it is sometimes still possible to reset a vertebrate CPG rhythm by activating an entire neural circuit for another behavior, thus demonstrating that the networks controlling these two behaviors either share key components or have strong interactions.

It has been shown previously that activation of a flexion reflex motor pattern can reset the walking rhythm in cats (Duysens, 1977b; Schomburg et al., 1998; McCrea, 2001) and scratching rhythms in turtles (Currie and Stein, 1989), consistent with shared circuitry for these behaviors. In addition, sensory stimulation that normally activates a flexion reflex can have phase-dependent effects during cat and human walking or walking rhythms (Forssberg et al., 1975, 1977; Andersson et al., 1978; Schomburg et al., 1978; Forssberg, 1979; Crenna and Frigo, 1984; Duysens et al., 1990; Degtyarenko et al., 1998; Burke, 1999; McCrea, 2001; Spaich et al., 2004; Sandrini et al., 2005; Duysens et al., 2013).

It has been unclear, however, whether cutaneous flexion reflex can also reset swimming rhythms. Lennard (1985) tested this hypothesis through electrical stimulation of leg cutaneous nerves in turtles during actual swimming movements and was unable to reset the swimming rhythm. Currie and Stein (1989) hypothesized, however, that to avoid activation of leg proprioceptors (which themselves reset the swim rhythm) during an actual flexion reflex movement, Lennard had to use cutaneous nerve stimuli that were too weak to reset the swim rhythm. Here, we tested the hypothesis that a stronger flexion-reflex-evoking foot skin stimulus can reset the swimming rhythm in turtles when movement-related sensory feedback is eliminated. We found that the flexion reflex stimulus often does reset the swim rhythm in a phase-dependent manner. In addition, the flexion reflex response to an electrical foot stim-
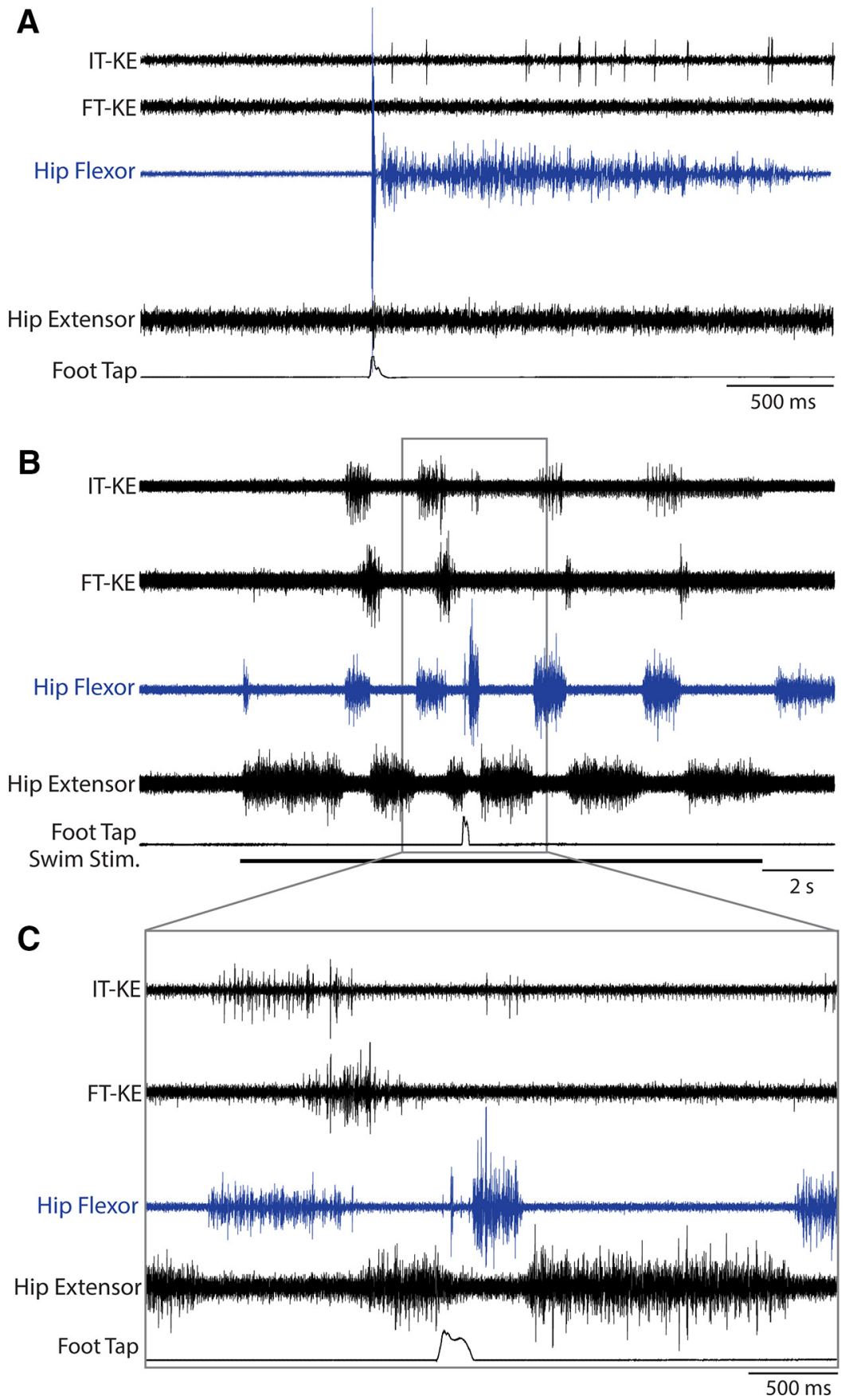

Figure 1. Examples of a typical fictive motor pattern evoked by a foot tap on its own $(\boldsymbol{A})$ and during the HE phase of fictive swimming $(\boldsymbol{B}, \boldsymbol{C})$ evoked by electrical stimulation of the contralateral lateral funiculus $(\boldsymbol{B})$ in a spinally transected, immobilized turtle. $\boldsymbol{B}$ shows the entire fictive swim episode. $($ expands the time at which the foot tap occurred (gray box in $\boldsymbol{B}$ ). Note that the foot tap quickly ended the HE burst, triggered a brief, high-amplitude HF burst at short latency, and caused a second, high-amplitude HF burst to occur soon afterward. IT-KE and FT-KE, iliotibialis and femorotibialis knee extensors, respectively.

ulus was itself altered in a phase-dependent manner. These findings demonstrate that the neural circuit for flexion reflex and the swimming CPG share key elements or have strong interactions between them.

\section{Materials and Methods}

Physiological methods. Physiological methods have been described previously (Lennard and Stein, 1977; Stein et al., 1982; Robertson et al., 1985; Currie and Stein, 1989; Berkowitz, 2001, 2002) and are summarized briefly here.

Animal preparations. Adult red-eared turtles $(n=6)$, Trachemys scripta elegans, of both sexes, weighing 350-800 g, were spinally tran- 


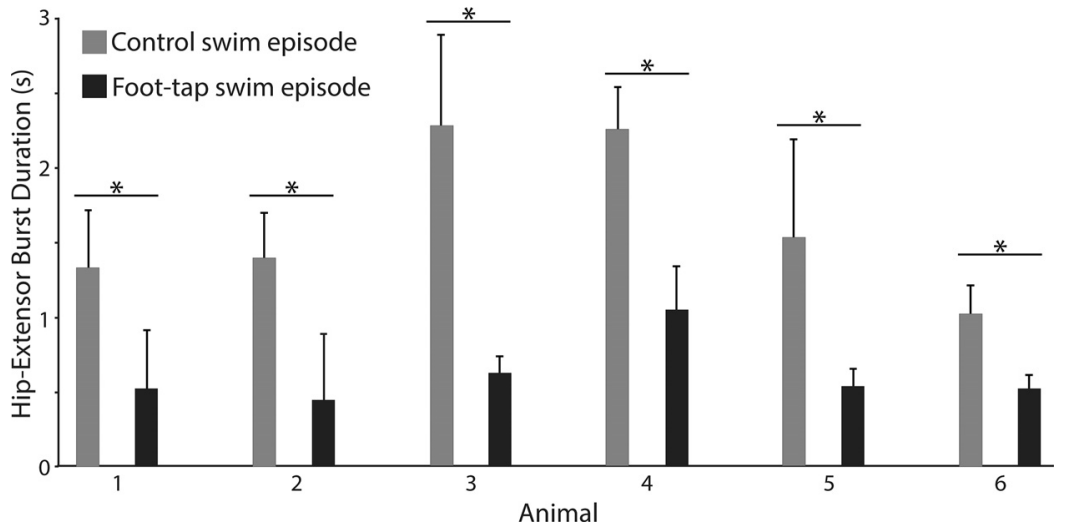

Figure 2. Swim HE bursts were shortened when a foot tap occurred during the fictive swim HE phase. Swim HE burst durations (mean \pm SD) were significantly greater during control fictive swimming (gray bars) than during a foot-tap cycle of fictive swimming (black bars) in all six animals tested. Asterisks indicate significant differences (Mann-Whitney test; $p<0.05$ ).

identical. Flexion reflex stimuli were delivered at arbitrary phases of fictive swimming (or fictive scratching), but the episodes were analyzed separately according to the phase in which the flexion reflex stimulus occurred.

Data analysis. Recordings were redigitized and analyzed quantitatively using Datapac (Run Technologies), rectified, and then smoothed with $100 \mathrm{~ms}$ time constant. The DC offset of each hip flexor nerve recording baseline was adjusted to zero as needed using Datapac. The onset and offset of each burst (i.e., a clear increase and then decrease in the nerve's rectified and smoothed amplitude) were determined in Datapac by positive and negative slope crossings using custom-selected thresholds. Cycle period was defined as the interval between two successive HF burst onsets. Burst duration was the interval between the onset and offset of a burst. Mean burst amplitude and integrated burst amplitude of HF were also

sected between the dorsal 2 (D2) and D3 roots under hypothermic analgesia (Robertson et al., 1985; Berkowitz, 2001). Several motor nerves were dissected free on the right (ipsilateral) side for extracellular recording: the hip flexor (HF), ventral puboischiofemoralis internus, pars anteroventralis; the hip extensor (HE), flexor cruris, pars flexor tibialis internus; and the knee extensors (KEs), triceps femoris, pars iliotibialis (IT-KE), pars ambiens (AM-KE), and/or pars femorotibialis (FT-KE) (Robertson et al., 1985). After surgery, turtles were allowed to warm to room temperature, immobilized with gallamine triethiodide $(8 \mathrm{mg} / \mathrm{kg}$, i.m.; Sigma-Aldrich), and artificially ventilated. All animal procedures were approved by the Institutional Animal Care and Use Committee of the University of Oklahoma.

Electrophysiology. Nerves were submerged in mineral oil surrounded by a wax well molded onto the turtle carapace. Each nerve's action potentials were recorded extracellularly using a pair of $100 \mu \mathrm{m}$ silver wires. Recordings were filtered (band-pass $0.1-1.0 \mathrm{kHz}$ ) and amplified $(\times 1000)$ using differential amplifiers (A-M Systems) and stored on a digital audio tape recorder (TEAC America).

Stimulation. Flexion reflex motor patterns (henceforth referred to as the fictive flexion reflex) were evoked by mechanical or electrical stimulation of the dorsal foot skin ipsilateral to the nerve recordings at a site that evoked the reflex strongly and reliably. The (immobilized) hindlimb was kept in an extended position in each experiment. The mechanical stimulus was a brief, gentle tap using a glass rod with a fire polished tip attached to a force transducer (Grass Technologies/Astro-Med) (Stein et al., 1982; Currie and Stein, 1989). The electrical stimulus was current passed between a pair of gold-plated disk electrodes (Grass Technologies) with their centers $\sim 1 \mathrm{~cm}$ apart that were affixed to the dorsal foot skin using electrode cream (Grass Technologies) (Currie and Stein, 1989; Berkowitz, 2007) either as a train of $51 \mathrm{~ms}$ pulses delivered at $100 \mathrm{~Hz}$ or a single $100 \mathrm{~ms}$ pulse $(4-90 \mathrm{~V})$. Forward-swimming motor patterns (henceforth referred to as fictive swimming) were evoked by electrical stimulation in the D3 contralateral lateral funiculus $(0.1 \mathrm{~ms}, 0.1-0.5 \mathrm{~mA}$ pulses at $40 \mathrm{~Hz}$ for $10-20 \mathrm{~s}$ ) using a pair of $100 \mu \mathrm{m}$ silver wires (California Fine Wire), insulated except at the tips, with one tip contacting the spinal cord and the other in the surrounding saline; such stimulation evokes unilateral swimming movements or motor patterns in the contralateral hindlimb (Lennard and Stein, 1977; Juranek and Currie, 2000; Berkowitz, 2002). Scratching motor patterns were evoked by continual gentle rubbing of a single site within the receptive field of one scratch form at $\sim 3-4 \mathrm{~Hz}$ using a glass probe with a fire-polished tip attached to a force transducer (Grass Technologies) (Stein et al., 1982; Mortin et al., 1985). Three minutes of rest was allowed between stimuli. To compare fictive swimming with and without mechanically evoked fictive flexion reflex, these two stimulation paradigms were alternated and the swim stimulation parameters were kept identical. Similarly, to compare electrically evoked fictive flexion reflex with and without fictive swimming (or with and without fictive scratching), these two stimulation paradigms were alternated and the flexion reflex stimulation parameters were kept determined in Datapac. Statistical analyses were performed using Instat 3 (GraphPad Software) with $p<0.05$ as the significance level.

\section{Results}

A turtle flexion-reflex motor pattern (Fig. $1 A$ ) typically includes a brief, short-latency, high-amplitude HF burst, followed after a pause of 20-30 ms by a seconds-long and gradually declining HF burst; the monoarticular knee extensor (FT-KE) is inactive during the HF burst (Stein et al., 1982; Currie and Stein, 1989; Currie and Lee, 1996). This is similar to the short-latency and longlatency components of cat flexion reflex (Sherrington, 1910). In a moving animal, the turtle flexion reflex retracts the limb into the hindlimb pocket, allowing the animal's shell to provide protection (Stein et al., 1982). A forward-swimming motor pattern (Fig. $1 B, C)$ typically includes long HE bursts alternating with shorter HF bursts. IT-KE bursts begin at about the same time as HF bursts, whereas FT-KE bursts are delayed; there is a gradual increase in cycle period during an episode (Lennard and Stein, 1977; Juranek and Currie, 2000; Berkowitz, 2002).

\section{Foot taps during fictive swimming HE bursts}

When we tapped the foot during the HE phase of fictive swimming, the HE burst quickly ended (Fig. $1 B, C$ ). The HF nerve (blue) in some cases displayed a brief, short-latency, high-amplitude burst such as typically occurs as the initial component of fictive flexion reflex elicited on its own (Fig. $1 A$ ), followed by a pause and then a second burst; this pause was typically longer $(\sim 100 \mathrm{~ms})$ than the usual pause between the first and second components of a fictive flexion reflex on its own (20-30 ms). The second HF burst was of higher amplitude than a typical swim HF burst and occurred without FT-KE activity, like a flexion reflex and unlike swimming, and began earlier than the next swim HF burst normally would. However, this second burst was briefer than either a typical flexion reflex or a typical swim HF burst. Therefore, this burst had characteristics that differed from both a flexion reflex and a swim motor pattern elicited on their own. We termed this a double-burst episode. In other cases (data not shown), it was not possible to discriminate two separate HF bursts; instead, there was a single, continuous HF burst that did not decline all the way to the baseline and that also often occurred earlier than the next fictive swim HF burst normally would. We termed this a merged-burst episode. For practical reasons, we separately analyzed double-burst and merged-burst episodes for some purposes (see below), although it appears that there was a continuum of responses to foot tap, with two separate HF bursts on 

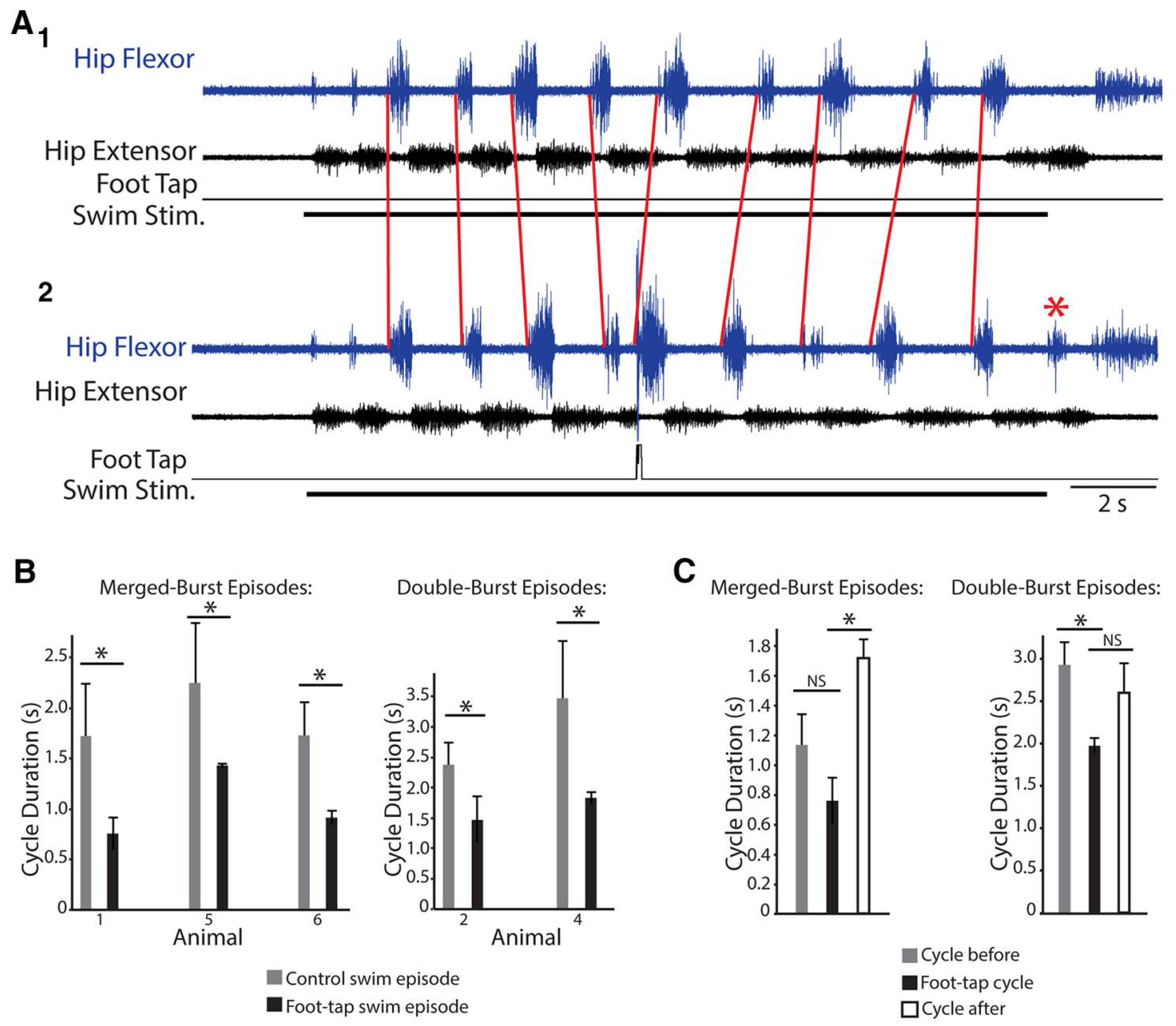

C

Merged-Burst Episodes: Double-Burst Episodes:
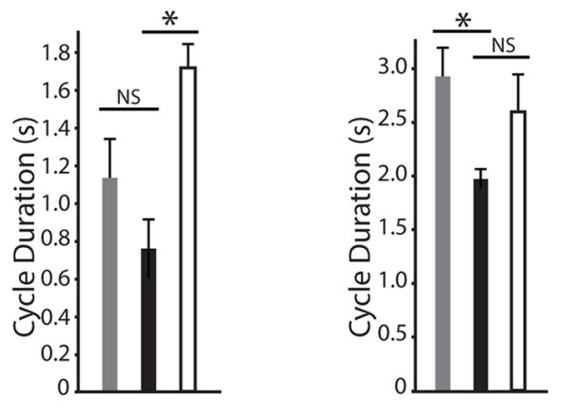

Figure 3. The fictive swim rhythm can be reset by a foot tap during the HE phase. $A$, Recordings from two episodes of fictive swimming in one animal are aligned by the timing of the swim stimulation (with identical stimulation parameters). Note that the upper (control, A1) fictive swim HF rhythm slightly led the lower (foot tap-episode, A2) HF rhythm until the foot tap occurred (red lines). After the foot tap, the lower HF rhythm consistently led the upper HF rhythm, showing that the fictive swim HF rhythm was reset by the foot tap and there was an extra HF burst at the end (asterisk). Across animals, the fictive swim cycle immediately after a foot tap was significantly briefer (means \pm SD; Mann-Whitney test, $p<0.05$ ) than fictive swim cycles from control episodes $(\boldsymbol{B})$ and often briefer than the fictive swim cycles just before and just after the foot-tap cycle within the same fictive swim episode (C).

one end of the spectrum and a completely merged HF burst on the other.

To assess quantitatively whether the fictive swim HE burst ended prematurely when the foot was tapped, we compared fictive swim HE burst durations during the foot-tap cycle to $\mathrm{HE}$ burst durations during control fictive swimming with no foot tap. For this purpose, we pooled cycles $2-5$ of control fictive swim episodes (which we alternated with foot-tap episodes), having found that these cycles generally did not differ significantly in cycle period (Kruskal-Wallis test, $p>0.05$ in 4/5 animals tested). In all six animals tested, the fictive swim HE burst duration was significantly shorter (Mann-Whitney test) during foot-tap cycles (Fig. 2, black bars) than during fictive swim control cycles (Fig. 2, gray bars). Therefore, the foot tap did end the fictive swim HE burst prematurely.

We compared HF burst durations after the foot tap separately for double-burst and merged-burst episodes. For each animal, the HF durations after the foot taps were compared with HF durations during control fictive swim episodes. A Mann-Whitney test was performed when the sample size for the animal was large enough. In foot-tap episodes with double bursts, the mean HF burst duration after the foot tap was significantly less than control fictive swim HF burst durations in three of three animals tested. In foot-tap episodes with merged bursts, the HF burst after the foot tap differed significantly in duration from control HF bursts in three of four animals tested; in two of these animals, the foot-tap HF burst was longer and in one it was shorter. In five of the six animals tested, the HF burst after the foot tap differed in duration from control fictive swim HF bursts for double-burst and/or merged-burst episodes.

The foot tap might, in principle, alter the HE and HF bursts during the foot-tap cycle alone or it may also alter the timing of subsequent cycles within the fictive swim episode. Therefore, we also examined the timing of subsequent fictive swim cycles, defined by the onset of each HF burst. Figure $3 A$ shows that a foot tap during a fictive swim HE burst could permanently alter the timing of subsequent cycles. In this figure, we have aligned (by the onset of swim stimulation) control-fictive swim (Fig. 3A1) and fictive swim + foot tap (Fig. 3A2) episodes from the same animal using the same swim stimulation parameters. In this case, the HF bursts (blue) from the foot-tap episode (Fig. 3A2) began slightly later than (i.e., lagged) the control-fictive swim HF bursts (Fig. $3 A 1)$ until the foot tap occurred; from this point forward, the foot-tap HF bursts occurred earlier than (i.e., led) the control- 


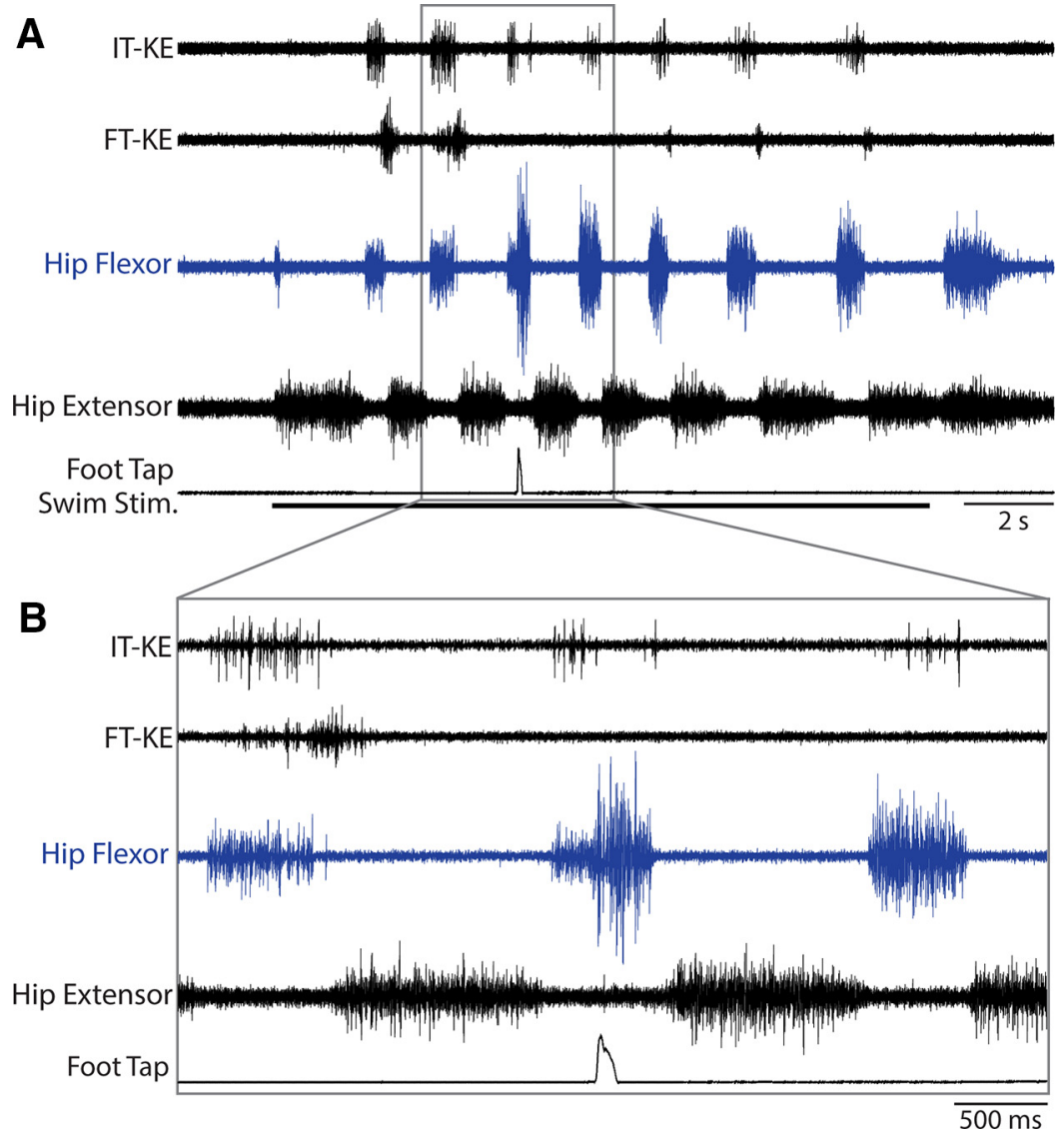

Figure 4. Example of a motor pattern evoked by a foot tap during the HF phase of fictive swimming. $\boldsymbol{B}$ expands the segment indicated by the gray box in $\boldsymbol{A}$. Note that the $\mathrm{HF}$ amplitude was increased, but there were no clear effects on the timing of either $\mathrm{HF}$ or HE bursts. IT-KE and FT-KE, iliotibialis and femorotibialis knee extensors, respectively.

fictive swim HF bursts (see red lines) and there was an extra HF burst at the end (asterisk). This shows that the timing of the fictive swim rhythm was permanently reset by the HE-phase foot tap in this episode, being phase advanced by the foot tap. This appeared to be a consistent effect across animals when the foot tap occurred during an HE burst.

We investigated quantitatively whether the fictive swim rhythm was reset by an HE-phase foot tap using two approaches separately for double-burst and merged-burst episodes; for double-burst episodes, we defined the foot-tap cycle to include both of the two HF bursts after the foot tap because it appeared that the brief first burst was an "extra" burst associated with the initial fictive flexion reflex response. First, we compared the duration (i.e., cycle period) of the cycles in which a foot tap occurred during the HE burst with cycles $2-5$ of control fictive swim episodes (Fig. 3B). We found that the foot-tap cycles were significantly shorter than control fictive swim cycles in all three animals that exhibited a sufficient number of merged burst episodes and in both animals that exhibited a sufficient number of doubleburst episodes. This shows that the fictive swim cycle with the HE-phase foot tap was shorter than normal, but if the subsequent fictive swim cycle was longer than normal, then the fictive swim rhythm might still return to its original timing. Therefore, we also compared statistically the duration of the fictive swim cycle immediately after the foot-tap cycle to the duration of control fictive swim cycles. We found that the fictive swim cycle after a HE-phase foot tap did not differ significantly in duration from control fictive swim cycles in 3 of 3 animals with suffi- cient sample size to test (Mann-Whitney test; $p=0.89,0.11$, and 0.23 ).

Second, we compared the duration of the foot-tap cycle to the durations of the cycles immediately before and after this cycle within the same fictive swim episode (Fig. 3C). We found that the foot-tap cycle was significantly shorter (Friedman's test followed by post hoc Dunn's test) than the previous cycle or shorter than the subsequent cycle in both merged-burst and double-burst episodes for the animal that yielded sufficient data for this test.

Therefore, the fictive swim cycle during which the foot tap occurred was shortened but subsequent fictive swim cycles in the same episode had normal durations. This demonstrates that the timing of fictive swim cycles was reset by the foot tap, such that each subsequent cycle began earlier than it would have without the foot tap. In other words, the flexion reflex stimulation during the HE phase reset the fictive swim rhythm by phase advancing the rhythm.

\section{Foot taps during fictive swimming HF bursts}

Foot taps had less dramatic and less consistent effects when they occurred during a fictive swim HF burst. The foot tap typically triggered an increase in amplitude and sometimes duration of the HF burst during which it occurred (Fig. 4). The duration of this HF burst differed significantly from control fictive swim HF burst durations in four of five animals with sufficient data to test this, but it increased in three of these animals and decreased in the other animal (Mann-Whitney test). There also appeared to be no consistent effect on the timing of subsequent fictive swim cycles (Fig. 4), in contrast to foot taps during HE bursts. In one trial in one animal and two trials in another animal, both the HF burst duration and the swim cycle duration clearly increased on the foot-tap cycle, but the next swim cycle was of normal duration, implying that the swim rhythm had been reset with a phase delay (data not shown); the foot tap had been early in the HF burst in one of these cases and approximately in the middle of the HF burst in the other two. To test the effect quantitatively across the entire dataset, we first compared the duration of the cycle in which the foot tap occurred during an HF burst with control fictive swim cycles. These differed significantly (MannWhitney test) in only two of five animals, with a significant increase in one animal and a significant decrease in the other. Second, we compared the duration of the cycle in which the foot tap occurred during a HF burst with the durations of the cycles immediately before and after this in the same fictive swim episode. In both of the animals for which there were enough data to perform this test, there was no significant difference in cycle duration. Therefore, the effect of foot tap was phase dependent: when it occurred during an HE burst, it often reset the fictive swim rhythm, but when it occurred during an HF burst, it had no consistent effect on subsequent fictive swim cycle timing. 
Electrical foot stimulation during fictive swimming

In addition to testing the effects of fictive flexion reflex on fictive swimming, we wished to test the effects of fictive swimming on fictive flexion reflex. For this purpose, we needed to use a foot stimulus that was too weak to reset the fictive swim rhythm and that, on its own, triggered a fictive flexion reflex of intermediate amplitude and duration so that we could detect an increase or decrease in fictive flexion reflex due to the ongoing swim. Therefore, we used an electrical stimulus to the foot skin and adjusted its amplitude in each animal to trigger an HF nerve response of intermediate amplitude and duration (Fig. 5A), less than that evoked by a tap to the foot (Fig. 1A).

We separately analyzed electrical foot stimuli that occurred during the HE and the HF phases of fictive swimming. Figure $5 A$ shows the HF response to an electrical foot stimulus on its own. Figure $5 B$ shows the identical stimulus in the same animal within a few minutes of the first stimulus when the stimulus occurred during a fictive swim HE burst. In this case, the HF fictive flexion reflex response was entirely eliminated, although there appeared to be a brief pause in the ongoing HE activity. For comparison, we tested whether a scratching motor pattern could also alter the HF fictive flexion reflex response (data not shown). Again, the HF fictive flexion reflex response was entirely eliminated, although there was a brief pause in the ongoing $\mathrm{HE}$ activity.

In general, the HF response to an electrical foot stimulus was reduced or eliminated if it occurred during a fictive swim $\mathrm{HE}$ burst. The HF fictive flexion reflex response was entirely eliminated in three of six animals tested; in two of the other three animals, it was significantly reduced in mean amplitude (MannWhitney test); in the remaining animal, the sample size was too small to perform the statistical test. Similarly, the HF response to an electrical foot stimulus was reduced or eliminated when it occurred during a pocket scratch HE burst. The response was entirely eliminated in three of four animals tested and was significantly reduced in mean amplitude in the one remaining animal (Mann-Whitney test).

The effects of swim on the response to an electrical foot stimulus were less obvious and less consistent when the stimulus occurred during an HF burst, showing a phase dependency similar to that seen for foot tap responses. The electrical foot stimulus typically evoked a small and brief $(<50 \mathrm{~ms})$ increase in the swim HF activity (data not shown), similar to the initial component of a flexion reflex on its own (Fig. 1A).

\section{Discussion}

The major finding of this study is that flexion-reflex-evoking stimulation of the foot skin can interrupt and reset the fictive forward swimming rhythm, phase advancing all subsequent swim cycles in the fictive swim episode. This demonstrates that the flexion reflex spinal circuit either shares key components with the swim CPG or has strong interactions with it. To our knowledge, this is the first demonstration that a leg flexion-reflexevoking stimulus can reset the swimming rhythm in any species.

Our finding contradicts Lennard's (1985) finding that electrical stimulation of a leg cutaneous nerve does not permanently reset the rhythm of turtle forward swimming movements. However, as noted by Currie and Stein (1989), Lennard used a weak stimulus that was subthreshold for a flexion reflex to avoid activating proprioceptive afferents that could themselves reset the swim rhythm; this may have prevented Lennard from detecting resetting caused by strong cutaneous stimulation. Our finding confirms the prediction of Currie and Stein that resetting of the fictive swim rhythm by foot stimulation would occur in an immobilized preparation. Weak foot stimuli might simply be below a threshold to trigger resetting. Alternatively, weak and strong stimuli might activate populations of cutaneous sensory neurons that promote opposite responses (hip extension and flexion, respectively) differentially, similar to what has been seen in cats (Sherrington and Sowton, 1911; Duysens and Pearson, 1976; Duysens, 1977a).

This finding adds to previous evidence that flexion reflex stimuli can reset the forward walking rhythm in cats (Duysens, 1977b; Schomburg et al., 1998; McCrea, 2001; Duysens et al., 2013) and all three forms of scratching rhythms in turtles (Currie and Stein, 1989), thus collectively showing that flexion reflex shares key components with or strongly interacts with CPGs for multiple forms of locomotion and scratching in limbed vertebrates generally. Although this kind of evidence is indirect, the simplest 
hypothesis consistent with these data is probably that there are hip-flexion-generating spinal interneurons shared among all of these behaviors (Lundberg, 1979; Schomburg et al., 1998; Burke, 1999; McCrea, 2001; Duysens et al., 2013). This hypothesis is consistent with previous findings that many spinal interneurons are activated during all three motor patterns: fictive flexion reflex, fictive locomotion, and fictive scratching (Berkowitz, 2002, 2005; Berkowitz et al., 2006). It is true that there are also fictive flexionreflex-selective interneurons that are inhibited during fictive locomotion and fictive scratching and that such interneurons can actually be most inhibited during the HF phase of fictive locomotion and fictive scratching (Berkowitz, 2007). However, it may be that shared, multifunctional interneurons are more numerous than flexion-reflex-selective interneurons and dominate interactions between these circuits such that sudden activation of flexion-phase interneurons is able to reset the locomotor and scratching CPGs.

Other hypotheses are also consistent with our data. One alternative hypothesis is that the flexion reflex circuit shares some components with locomotor CPGs and other components with scratching CPGs. Some previous data are consistent with this hypothesis: there are some spinal interneurons activated during both fictive scratching and fictive flexion reflex, but not fictive swimming (Berkowitz, 2002). Another alternative hypothesis is that the interneurons necessary for generation of flexion reflex are not members of the locomotor or scratch CPGs, but nonetheless have sufficiently strong interactions with those CPGs to reset them. Although possible, this hypothesis leads to the question of whether a neuron that has such a strong effect on a CPG should be considered to be outside of the CPG (Hao et al., 2011).

We also found here that a flexion-reflex-evoking stimulus was more likely to reset the fictive swim rhythm if it occurred during the fictive swim HE phase, as opposed to the fictive swim HF phase, and that HE-phase resets were always phase advances, as has been found previously for cat walking rhythms (Schomburg et al., 1998) and turtle scratching rhythms (Currie and Stein, 1989). In addition, the HF response to a weak flexion-reflexevoking stimulus was reduced or eliminated if it occurred during the fictive swim (or fictive scratch) HE phase, but had less consistent effects if it occurred during the fictive swim (or fictive scratch) HF phase. Therefore, the effects of the foot stimulus were phase dependent during fictive swimming.

This finding adds to previous evidence of phase-dependent effects of flexion reflex stimuli during forward and backward walking rhythms, especially from studies of cats and humans (Forssberg et al., 1975, 1977; Andersson et al., 1978; Schomburg et al., 1978; Forssberg, 1979; Wand et al., 1980; Crenna and Frigo, 1984; Buford and Smith, 1990; Duysens et al., 1990; Duysens et al., 1992; Duysens et al., 1996; Degtyarenko et al., 1998; Burke, 1999; Ménard et al., 1999; Perreault et al., 1999; McCrea, 2001; Spaich et al., 2004; Quevedo et al., 2005; Sandrini et al., 2005; Hultborn, 2006; Knikou et al., 2006; Knikou, 2007; Knikou et al., 2009; Duysens et al., 2013) and during swimming (Lennard, 1985) and all three forms of scratching rhythms in turtles (Currie and Stein, 1989). Collectively, these findings suggest that leg flexion reflex (as well as other leg reflexes) are modulated or gated according to hip phase for multiple forms of locomotion and scratching in limbed vertebrates generally. In addition, an axial flexion reflex is gated out via phasic inhibition of sensory interneurons during hatchling tadpole axial fictive swimming (Sillar and Roberts, 1988, 1992). Phase-dependent inhibition of sensory reflexes during rhythmic motor patterns has also been demonstrated in other vertebrates and in several invertebrates (Clarac et al., 1992; Clarac et al., 2000).

Modulation of flexion reflexes serves clear purposes during walking. If not reduced during the hip extension phase, then the flexion reflex could undermine an animal's stability if the animal is relying on extension of that leg to support its weight (McCrea, 2001). In addition, accentuation of flexion reflex during the hip flexion phase may help the animal avoid obstacles contacted by its leg (Forssberg, 1979; Wand et al., 1980; Buford and Smith, 1993; McCrea, 2001; Quevedo et al., 2005). It is less clear why such flexion reflex modulation would be necessary during swimming, because the animal does not rely on leg extension for weight support during swimming. One explanation is that a flexion reflex during the power stroke (HE phase) of forward swimming would be disruptive to the animal's ability to propel itself forward, which could be very important when the animal is escaping from a predator, for example. A second explanation is that largely shared circuits of multifunctional spinal interneurons for flexion reflex, locomotion, and scratching evolved across limbed vertebrates for other functional reasons that are unrelated to the effects of foot stimulation on swimming or vice versa.

The potency of a flexion reflex stimulus to reset the fictive swimming rhythm during the HE phase, but not the HF phase, may also point to the primacy of HF-timed interneurons in spinal rhythm generation. This kind of asymmetry has been seen previously in studies of spinal central pattern generation in turtles, rodents, and cats (Duysens et al., 2013). For example, HE deletions are much more common than HF deletions in turtle and cat fictive scratching (Robertson et al., 1985; Lafreniere-Roula and McCrea, 2005); the HF rhythm typically continues during these HE deletions in turtle rostral scratching (Robertson and Stein, 1988) and HF-timed interneurons continue to fire rhythmically (Stein and Daniels-McQueen, 2002). In addition, rhythmic flexor motoneuron activity continues during non-resetting extensor deletions in mouse fictive locomotion, but extensor motoneuron activity typically becomes tonic during flexor deletions (Zhong et al., 2012).

\section{References}

Andersson O, Forssberg H, Grillner S, Lindquist M (1978) Phasic gain control of the transmission in cutaneous reflex pathways to motoneurones during 'fictive' locomotion. Brain Res 149:503-507. CrossRef Medline

Berkowitz A (2001) Broadly tuned spinal neurons for each form of fictive scratching in spinal turtles. J Neurophysiol 86:1017-1025. Medline

Berkowitz A (2002) Both shared and specialized spinal circuitry for scratching and swimming in turtles. J Comp Physiol A Neuroethol Sens Neural Behav Physiol 188:225-234. CrossRef Medline

Berkowitz A (2005) Physiology and morphology indicate that individual spinal interneurons contribute to diverse limb movements. J Neurophysiol 94:4455-4470. CrossRef Medline

Berkowitz A (2007) Spinal interneurons that are selectively activated during fictive flexion reflex. J Neurosci 27:4634-4641. CrossRef Medline

Berkowitz A (2010) Multifunctional and specialized spinal interneurons for turtle limb movements. Ann N Y Acad Sci 1198:119-132. CrossRef Medline

Berkowitz A, Yosten GL, Ballard RM (2006) Somato-dendritic morphology predicts physiology for neurons that contribute to several kinds of limb movements. J Neurophysiol 95:2821-2831. CrossRef Medline

Berkowitz A, Roberts A, Soffe SR (2010) Roles for multifunctional and specialized spinal interneurons during motor pattern generation in tadpoles, zebrafish larvae, and turtles. Front Behav Neurosci 4:36. Medline

Briggman KL, Kristan WB (2008) Multifunctional pattern-generating circuits. Annu Rev Neurosci 31:271-294. CrossRef Medline

Brown TG (1911) The intrinsic factors in the act of progression in the mammal. Proc R Soc London 84:308-319. CrossRef

Buford JA, Smith JL (1990) Adaptive control for backward quadrupedal walking. II. Hindlimb muscle synergies. J Neurophysiol 64:756-766. Medline 
Buford JA, Smith JL (1993) Adaptive control for backward quadrupedal walking. III. Stumbling corrective reactions and cutaneous reflex sensitivity. J Neurophysiol 70:1102-1114. Medline

Burke RE (1999) The use of state-dependent modulation of spinal reflexes as a tool to investigate the organization of spinal interneurons. Exp Brain Res 128:263-277. CrossRef Medline

Clarac F, el Manira A, Cattaert D (1992) Presynaptic control as a mechanism of sensory-motor integration. Curr Opin Neurobiol 2:764-769. CrossRef Medline

Clarac F, Cattaert D, Le Ray D (2000) Central control components of a 'simple' stretch reflex. Trends Neurosci 23:199-208. CrossRef Medline

Crenna P, Frigo C (1984) Evidence of phase-dependent nociceptive reflexes during locomotion in man. Exp Neurol 85:336-345. CrossRef Medline

Currie SN, Lee S (1996) Glycinergic inhibition in the turtle spinal cord regulates the intensity and pattern of fictive flexion reflex motor output. Neurosci Lett 205:75-78. CrossRef Medline

Currie SN, Stein PS (1989) Interruptions of fictive scratch motor rhythms by activation of cutaneous flexion reflex afferents in the turtle. J Neurosci 9:488-496. Medline

Degtyarenko AM, Simon ES, Norden-Krichmar T, Burke RE (1998) Modulation of oligosynaptic cutaneous and muscle afferent reflex pathways during fictive locomotion and scratching in the cat. J Neurophysiol 79: 447-463. Medline

Duysens J (1977a) Reflex control of locomotion as revealed by stimulation of cutaneous afferents in spontaneously walking premammillary cats. J Neurophysiol 40:737-751. Medline

Duysens J (1977b) Fluctuations in sensitivity to rhythm resetting effects during the cat's step cycle. Brain Res 133:190-195. CrossRef Medline

Duysens J, Pearson KG (1976) The role of cutaneous afferents from the distal hindlimb in the regulation of the step cycle of thalamic cats. Exp Brain Res 24:245-255. Medline

Duysens J, Trippel M, Horstmann GA, Dietz V (1990) Gating and reversal of reflexes in ankle muscles during human walking. Exp Brain Res 82: 351-358. Medline

Duysens J, Tax AA, Trippel M, Dietz V (1992) Phase-dependent reversal of reflexly induced movements during human gait. Exp Brain Res 90: 404-414. Medline

Duysens J, Tax AA, Murrer L, Dietz V (1996) Backward and forward walking use different patterns of phase-dependent modulation of cutaneous reflexes in humans. J Neurophysiol 76:301-310. Medline

Duysens J, De Groote F, Jonkers I (2013) The flexion synergy, mother of all synergies and father of new models of gait. Front Comput Neurosci 7:14. Medline

Forssberg H (1979) Stumbling corrective reaction: a phase-dependent compensatory reaction during locomotion. J Neurophysiol 42:936-953. Medline

Forssberg H, Grillner S, Rossignol S (1975) Phase dependent reflex reversal during walking in chronic spinal cats. Brain Res 85:103-107. CrossRef Medline

Forssberg H, Grillner S, Rossignol S (1977) Phasic gain control of reflexes from the dorsum of the paw during spinal locomotion. Brain Res 132: 121-139. CrossRef Medline

Hao ZZ, Spardy LE, Nguyen EB, Rubin JE, Berkowitz A (2011) Strong interactions between spinal cord networks for locomotion and scratching. J Neurophysiol 106:1766-1781. CrossRef Medline

Hultborn H (2006) Spinal reflexes, mechanisms and concepts: from Eccles to Lundberg and beyond. Prog Neurobiol 78:215-232. CrossRef Medline

Jankowska E, Jukes MG, Lund S, Lundberg A (1967) The effect of DOPA on the spinal cord. 6. Half-centre organization of interneurones transmitting effects from the flexor reflex afferents. Acta Physiol Scand 70:389-402. CrossRef Medline

Juranek J, Currie SN (2000) Electrically evoked fictive swimming in the lowspinal immobilized turtle. J Neurophysiol 83:146-155. Medline

Knikou M (2007) Hip-phase-dependent flexion reflex modulation and expression of spasms in patients with spinal cord injury. Exp Neurol 204: 171-181. CrossRef Medline

Knikou M, Kay E, Rymer WZ (2006) Modulation of flexion reflex induced by hip angle changes in human spinal cord injury. Exp Brain Res 168:577-586. CrossRef Medline

Knikou M, Angeli CA, Ferreira CK, Harkema SJ (2009) Flexion reflex modulation during stepping in human spinal cord injury. Exp Brain Res 196: 341-351. CrossRef Medline

Lafreniere-Roula M, McCrea DA (2005) Deletions of rhythmic motoneu- ron activity during fictive locomotion and scratch provide clues to the organization of the mammalian central pattern generator. J Neurophysiol 94:1120-1132. CrossRef Medline

Lennard PR (1985) Afferent perturbations during "monopodal" swimming movements in the turtle: phase-dependent cutaneous modulation and proprioceptive resetting of the locomotor rhythm. J Neurosci 5:1434-1445. Medline

Lennard PR, Stein PS (1977) Swimming movements elicited by electrical stimulation of turtle spinal cord. I. Low-spinal and intact preparations. J Neurophysiol 40:768-778. Medline

Lundberg A (1979) Multisensory control of spinal reflex pathways. Prog Brain Res 50:11-28. CrossRef Medline

Marder E, Calabrese RL (1996) Principles of rhythmic motor pattern generation. Physiol Rev 76:687-717. Medline

McCrea DA (2001) Spinal circuitry of sensorimotor control of locomotion. J Physiol 533:41-50. CrossRef Medline

Ménard A, Leblond H, Gossard JP (1999) The modulation of presynaptic inhibition in single muscle primary afferents during fictive locomotion in the cat. J Neurosci 19:391-400. Medline

Mortin LI, Keifer J, Stein PS (1985) Three forms of the scratch reflex in the spinal turtle: movement analyses. J Neurophysiol 53:1501-1516. Medline

Perreault MC, Shefchyk SJ, Jimenez I, McCrea DA (1999) Depression of muscle and cutaneous afferent-evoked monosynaptic field potentials during fictive locomotion in the cat. J Physiol 521:691-703. CrossRef Medline

Quevedo J, Stecina K, Gosgnach S, McCrea DA (2005) Stumbling corrective reaction during fictive locomotion in the cat. J Neurophysiol 94:2045-2052. CrossRef Medline

Robertson GA, Stein PS (1988) Synaptic control of hindlimb motoneurones during three forms of the fictive scratch reflex in the turtle. J Physiol 404:101-128. CrossRef Medline

Robertson GA, Mortin LI, Keifer J, Stein PS (1985) Three forms of the scratch reflex in the spinal turtle: central generation of motor patterns. J Neurophysiol 53:1517-1534. Medline

Sandrini G, Serrao M, Rossi P, Romaniello A, Cruccu G, Willer JC (2005) The lower limb flexion reflex in humans. Prog Neurobiol 77:353-395. CrossRef Medline

Schomburg ED, Meinck HM, Haustein J, Roesler J (1978) Functional organization of the spinal reflex pathways from forelimb afferents to hindlimb motoneurones in the cat. Brain Res 139:21-33. CrossRef Medline

Schomburg ED, Petersen N, Barajon I, Hultborn H (1998) Flexor reflex afferents reset the step cycle during fictive locomotion in the cat. Exp Brain Res 122:339-350. CrossRef Medline

Sherrington CS (1906) The integrative action of the nervous system. New York: Charles Scribner.

Sherrington CS (1910) Flexion-reflex of the limb, crossed extension-reflex, and reflex stepping and standing. J Physiol 40:28-121. CrossRef Medline

Sherrington CS, Sowton SCM (1911) Reversal of the reflex effect of an afferent nerve by altering the character of the electrical stimulus applied. Proc R Soc Lond B 83:435-446. CrossRef

Sillar KT, Roberts A (1988) A neuronal mechanism for sensory gating during locomotion in a vertebrate. Nature 331:262-265. CrossRef Medline

Sillar KT, Roberts A (1992) Phase-dependent modulation of a cutaneous sensory pathway by glycinergic inhibition from the locomotor rhythm generator in $\mathrm{Xe}$ nopus embryos. Eur J Neurosci 4:1022-1034. CrossRef Medline

Spaich EG, Arendt-Nielsen L, Andersen OK (2004) Modulation of lower limb withdrawal reflexes during gait: a topographical study. J Neurophysiol 91:258-266. Medline

Stein PS, Daniels-McQueen S (2002) Modular organization of turtle spinal interneurons during normal and deletion fictive rostral scratching. J Neurosci 22:6800-6809. Medline

Stein PSG, Robertson GA, Keifer J, Grossman ML, Berenbeim JA, Lennard PR (1982) Motor neuron synaptic potentials during fictive scratch reflex in turtle. J Comp Physiol 146:401-409. CrossRef

Wand P, Prochazka A, Sontag KH (1980) Neuromuscular responses to gait perturbations in freely moving cats. Exp Brain Res 38:109-114. Medline

Zhong G, Shevtsova NA, Rybak IA, Harris-Warrick RM (2012) Neuronal activity in the isolated mouse spinal cord during spontaneous deletions in fictive locomotion: insights into locomotor central pattern generator organization. J Physiol 590:4735-4759. CrossRef Medline 